\title{
ASSESSMENT OF LYMPHEDEMA WITH LYMPHOSCINTIGRAPHY: CAN NODAL QUANTIFICATION HELP?
}

\author{
N. Sampathirao, M. Indirani, G. Manokaran, A. Jaykanth, A. Patel, S. Simon
}

Departments of Nuclear Medicine and PET/CT (NS,MI,AJ,AP,SS), and Plastic Surgery (GM), Apollo Hospitals, Chennai, Tamil Nadu, India

\section{ABSTRACT}

Lymphoscintigraphy with combined qualitative and quantitative analysis is reported to be a more sensitive approach to diagnose lymphedema in comparison with the conventional clinical analysis. Our study seeks to evaluate the diagnostic performance of lower limb lymphoscintigraphy with amalgamation of qualitative and quantitative analysis by measuring the ilio-inguinal nodal uptake. This prospective observational study was comprised of 86 patients (172 limbs) diagnosed with lower limb lymphedema. After a thorough clinical grading of edema, radionuclide lymphoscintigraphy was performed as per a dedicated institutional protocol. Ilio-inguinal nodal quantification of tracer uptake was computed along with the visual study of the scans. Additionally, the corresponding mean nodal uptake percentage for each grade of lymphedema was assessed and a cut off nodal uptake percentage to differentiate between normal and abnormal limbs was defined. Although quantitative analysis with nodal uptake percentage provides objective criteria to diagnose lymphedema, it can only act as an adjunct to qualitative method without replacing it. Finally, standardization of procedure for quantitative lymphoscintigraphy is needed including the potential for combining both rate of clearance of tracer from injection site and nodal uptake for quantification.

Keywords: lymphoscintigraphy, quantitative lymphoscintigraphy, ilio-inguinal nodal uptake percentage, lymphedema, staging, filariasis

Lymphoscintigraphy has become a key investigation to diagnose lymphedema and visualize the lymphatic system. It has largely replaced the more invasive and technically difficult technique of lymphangiography which includes invasive methodology (1). Moreover, the iodized lipiodol is no longer freely available and in recent times of radionuclide lymphoscintigraphy and MR lymphangiography, it has almost become obsolete. There is no internationally agreed standardized technique for lymphoscintigraphy and thus results are varied and difficult to compare. Major differences occur in the radiotracers used, sites of injection, different imaging protocols, and methods of analysis (2). Analysis is done by visual qualitative method, which is subjective and has a wider inter-observer variability. Subtle differences in ilio-inguinal uptake between normal and abnormal limbs may be missed with qualitative analysis (2).

The addition of quantitative analysis to interpret lymphoscintigraphy has been shown to increase the sensitivity and specificity of the scan in diagnosing lymphedema. Parameters including lymph nodal uptake and rate of clearance of tracer from injection site are employed for quantifying the degree of lymphedema (2). However, standardization of methods, establishment of normal values, 
and definition of abnormalities are needed. In addition, in cases of unilateral lymphedema, the contralateral limb can be found to be abnormal (3). In such a scenario, where the contralateral limb may appear normal clinically, lymphoscintigraphy with visual analysis and quantification of lymphatic dysfunction often help to elicit subclinical lymphedema (3). Some prior studies have undertaken quantitative analysis of lymphoscintigraphy, yet quantification could not be standardized due to varied and cumbersome study protocols, and methods of quantification.

In this study, the method of quantification undertaken is to measure the accumulation of radiotracer in the nodes by calculating the nodal uptake percentage (4). This study extends its interest to quantify the degree of abnormality corresponding to different grades of edema as stated by qualitative analysis and also to detect subclinical lymphedema in the contralateral limb in patients presenting with unilateral limb lymphedema where early intervention can significantly reduce the morbidity.

\section{MATERIALS AND METHOD}

This prospective observational diagnostic study was carried out in the Department of Nuclear Medicine and PET/CT in Apollo hospitals, Chennai over a period of 2 years from May 2017 till May 2019. The study included 9 controls (18 limbs) - 4 males and 5 females and 86 patients (172 limbs), 37 females and 49 males in the age range of 18-78 years, diagnosed with lower limb lymphedema after excluding other causes of edema.

Out of 86 patients, 3 were diagnosed with primary lymphedema, 6 had history of recurrent cellulitis out of which 4 were chronic diabetics, 5 displayed chronic venous insufficiency, 10 had a history of trauma to the leg, 15 cases were treated for filariasis which was proved with filarial antigen detection. 17 patients were from endemic areas of filarial infections and thus had received empirical anti-filarial treatment. The cause of lymphedema in rest of the 30 patients was not known. $66 / 86$ patients had bilateral lymphedema and $20 / 86$ presented with unilateral limb edema. Before being enrolled in this study, a written informed consent for participation in this study was taken from all patients in accordance with the regulations of the institutional review board which approved the study. Careful history, physical examination and review of previous ancillary investigations were performed. Patients underwent lower limb lymphoscintigraphy with Tc99m-Antimony sulfide using a designated standardized institutional protocol detailed below.

\section{Selection Criteria}

Controls: As this was not a case control study, controls were selected arbitrarily. Only 9 controls had volunteered for the study. Controls were all above 18 years of age (ranging from 20-45 years) with no history of lower limb edema or other comorbidities and were enrolled after obtaining informed consent to the study.

Cases: Patients of age 18 years and above with either congenital or acquired, unilateral or bilateral lower limb lymphedema of any clinical grading were included, age ranging from 18-78 years. Patients with cardiac, hepa-tic, renal comorbidities, or a history of vascular diseases in the lower limbs were excluded. In case of acute infection of inflammation/cellulitis of the limb, lymphoscintigraphy was performed after antibiotic administration or later on resolution of the inflammation.

Exclusion criteria: Patients or controls with prior nodal surgery or radiotherapy to pelvic region were excluded from the study groups.

\section{Clinical Assessment}

Clinical grading of the limb was done prior to the scan according to the International Society of Lymphology guidelines (5) (Table 1 and Figs. 1a, b, $c, d$ ).

\section{Imaging}

Preparation and radiopharmaceutical 


\section{TABLE 1 \\ Clinical Grading of Lymphedema Utilized for Study (5) (See Fig. 1 a,b,c,d)}

Grade 0: No apparent edema/subclinical lymphedema

Grade I: Pitting edema of the limb, completely reversible on elevation

Grade II: Non pitting edema of the limb, does not reduce completely on elevation

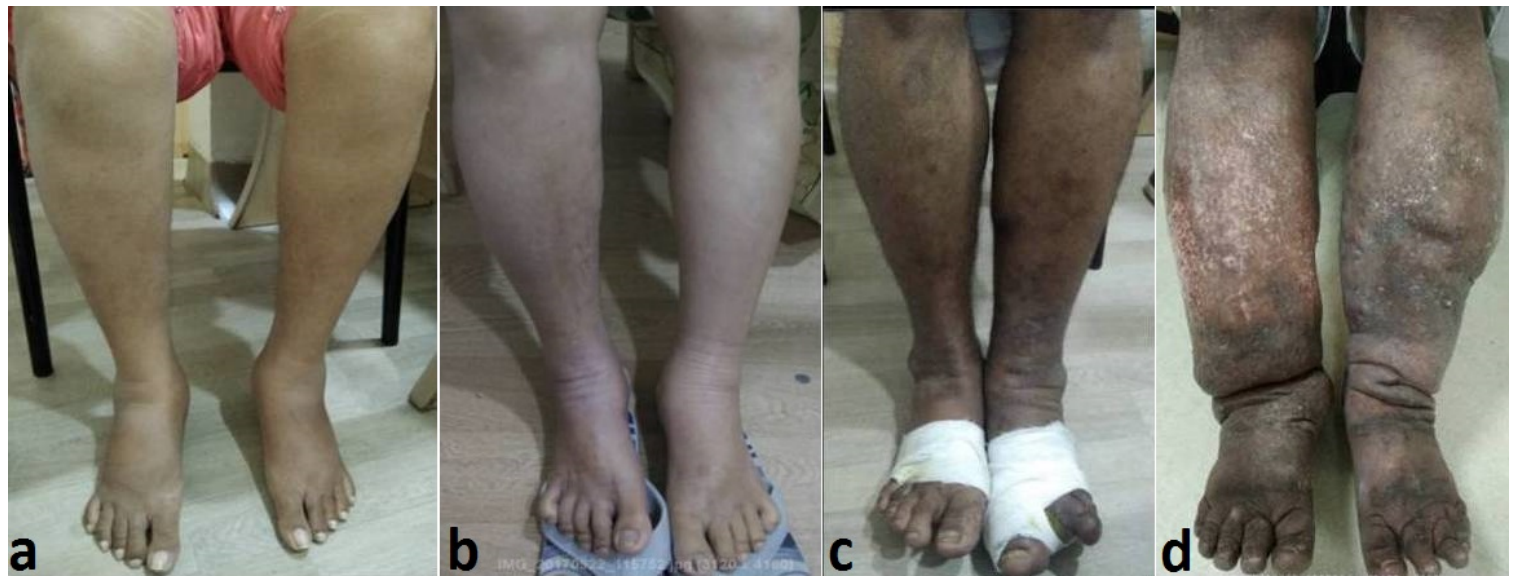

Fig. 1 (a-d). Clinical grading of lymphedema. a) Grade 0: no apparent edema b) Grade I: pitting edema of the limb, but completely reversible on elevation c) Grade II: non pitting edema, does not reduce completely on elevation d) Grade III: non pitting, non-reversible edema of the limb with skin changes.

Patient counseling about the route of injection and duration of the scan was done. No fasting was required. Tc99m-antimony sulfide was utilized for imaging.

Patient was positioned supine on the table with the head facing outwards. A single injection of $0.2 \mathrm{ml}$ containing $0.5 \mathrm{mCi}$ of Tc99m-antimony sulfide was given intradermally in the dorsum of each foot between the $1^{\text {st }}$ interdigital space simultaneously on both limbs by two personnel under sterile aseptic precautions.

\section{Acquisition}

Immediate, post exercise, and delayed at 3 hours sweep studies from the level of D12 to the feet were acquired at the rate of $10 \mathrm{~cm}$ per minute in anterior and posterior views using $256 \times 1024$ pixels of a large field of view gamma camera (Symbia T6) with dual head system and LEAP collimators. The mode of exercise was flapping of the feet on the table for the duration of the sweep study. 24 hours delayed images were also taken in 6 patients where inguinal nodes were not visualized on 3 $\mathrm{hr}$ delayed images. The same procedure was used for the control.

\section{Analysis}

\section{Qualitative assessment}

Interpretation and staging was done by visual inspection of the observed patterns such as delay to flow, visualization of lymph nodes, 


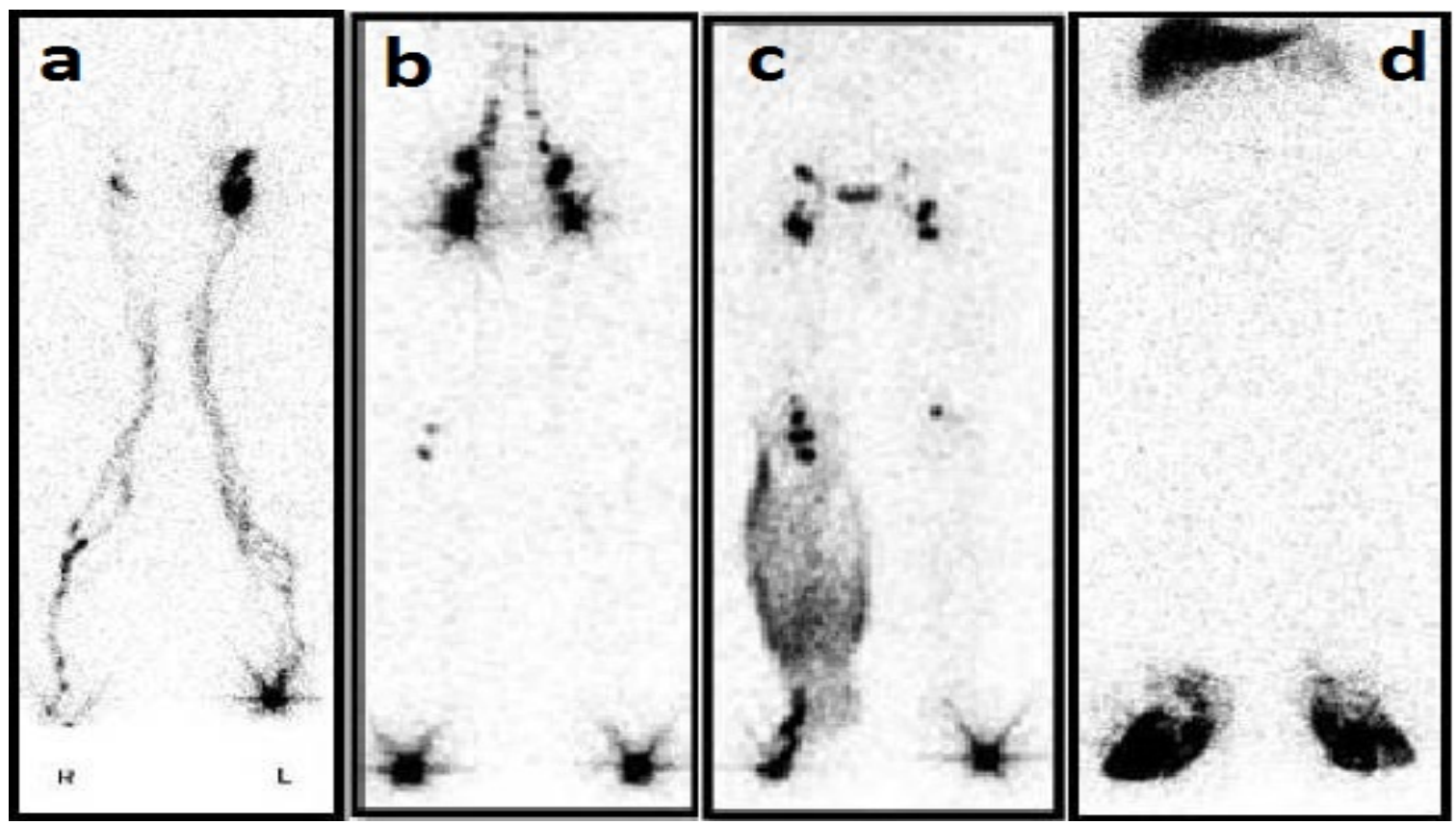

Fig. 2 (a-d). Qualitative grading based on lymphoscintigraphy. a) multiple or dilated lymphatic channels or delay to visualize lymph nodes in immediate images b) findings of Grade I and visualization of deep nodes (popliteal nodes) c) Tracer stasis or dermal backflow in delayed images, nodes visualized in delayed images d) Grades I, II and III with non-visualization of lymph nodes.

\section{TABLE 2}

\section{Grading of Lymphedema on Lymphoseintigraphy by Visual Analysis}

Grade I - Multiple or dilated lymphatic channels or delay to visualize lymphn odes in immediate images

Grade II - In addition to Grade I findings, flow through deeper lymphatic system(popliteal lymph nodes)

Grade III - Tracer stasis or dermal backflow in the delayed images. Lymph nodes visualized in delayed images

Grade IV - Grade I, II and III with non visualization of lymph nodes

flow through deeper lymphatic system, and dermal back flow. Patients were classified into four grades according to the scintigraphic grading system standardized at our institution (6) (Fig. 2, Table 2)

\section{Quantitative assessment}

For quantification of nodal uptake, 4 ROIs were drawn, 2 around all the visualized nodes and 2 around the injection sites in each limb (Fig. 3) in the 3 hour delayed images for both controls and patients. Gamma counts of the nodes (Gn) and injection site (Gi) were corrected to background and number of pixels. 


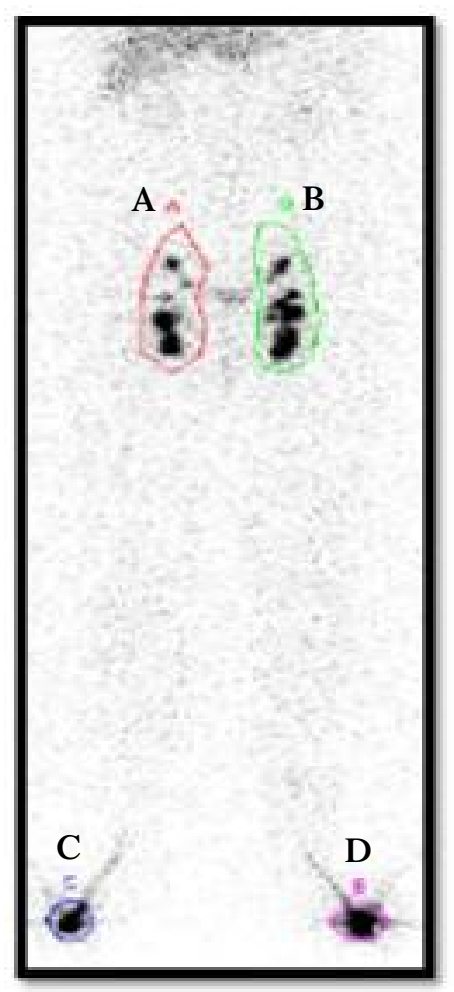

Fig. 3. ROIs for quantification of lymph nodal uptake percentage in "3 hour delayed images". A: ROI of right sided visualized nodes, B: ROI of left sided visualized nodes, $C$ : ROI of right injection site, $D:$ ROI of left injection site.

Nodal uptake percentage was calculated according to the following formula (4)

\section{NODAL UPTAKE \% $=(\mathrm{Gn} X 100) / \mathrm{Gi}+\mathrm{Gn}$}

Finally clinical, qualitative and quantitative assessment were tabulated and analyzed. Nodal uptake was represented as percentages and correlated with the corresponding scintigraphic visual grading. The difference between percentage uptake in visual grades was tested using one way ANOVA. All p values $<0.05$ were considered as statistically significant. A receiver operative curve analysis (ROC) was undertaken to define a cut-off nodal uptake percentage to differentiate normal from abnormal limbs using qualitative assessment with visual inspection as gold standard. The data initially were not distribut- ed normally. A logarithmic transformation was done following which the data were distributed normally and taking antilogarithms, the values were calculated.

\section{RESULTS}

Of 86 patients, $12 / 86(14 \%)$ patients had undergone surgical intervention for lymphedema either in the form of skin grafting for ulcers or debridement surgery for cellulitis. However, no history of lymphadenectomy was present.

\section{Analysis of percentage ilio-inguinal nodal uptake in controls}

A total of 9 controls (18 limbs) were analyzed. Clinical assessment and visual interpretation of scintigraphy showed no evidence of lymphedema. All control limbs showed a normal pattern of lymphatic flow on lymphoscintigraphy. The control group was then assessed by quantitative lymphoscintigraphy to calculate nodal uptake. The mean inguinal nodal uptake percentage was $36.52 \%$ with a standard deviation (SD) of 8.4. The range of nodal uptake was 19.72 to $53.32 \%$.

\begin{tabular}{|c|c|c|}
\hline \multicolumn{3}{|c|}{ TABLE 3 } \\
Nodal Uptake Percentage in Controls \\
\hline Sr. No. & $\begin{array}{c}\text { Right lower } \\
\text { limb (\%) }\end{array}$ & $\begin{array}{c}\text { Left lower } \\
\text { limb (\%) }\end{array}$ \\
\hline 1 & 39.87 & 28.25 \\
\hline 2 & 27.81 & 25.70 \\
\hline 3 & 30.97 & 27.25 \\
\hline 4 & 49.59 & 50.73 \\
\hline 5 & 45.58 & 46.56 \\
\hline 6 & 27.19 & 29.80 \\
\hline 7 & 39.51 & 41.63 \\
\hline 8 & 44.67 & 35.64 \\
\hline 9 & 32.69 & 33.94 \\
\hline
\end{tabular}




\begin{tabular}{|c|c|}
\hline \multicolumn{2}{|c|}{ TABLE 4} \\
\hline \multicolumn{2}{|c|}{$\begin{array}{l}\text { a. Limb Distribution According to } \\
\text { Clinical Grades }\end{array}$} \\
\hline Clinical grade & $\begin{array}{l}\text { Number } \\
\text { of limbs }\end{array}$ \\
\hline 0 & 45 \\
\hline 1 & 62 \\
\hline 2 & 21 \\
\hline 3 & 44 \\
\hline \multicolumn{2}{|c|}{$\begin{array}{l}\text { b. Limb Distribution According } \\
\text { to Qualitative Grading }\end{array}$} \\
\hline $\begin{array}{c}\text { Qualitative } \\
\text { lymphoscintigraphic } \\
\text { grade }\end{array}$ & $\begin{array}{l}\text { Number } \\
\text { of limbs }\end{array}$ \\
\hline Normal & 19 \\
\hline Grade I & 80 \\
\hline Grade II & 24 \\
\hline Grade III & 45 \\
\hline Grade IV & 4 \\
\hline
\end{tabular}

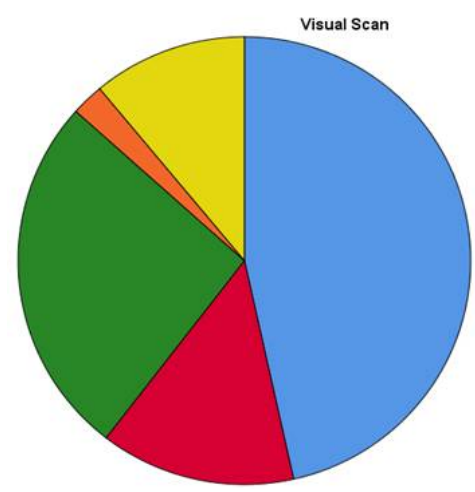

Fig. 4. Distribution of limbs based on qualitative analysis.

Analysis of patients with lymphoscintigraphy by qualitative and quantitative methods

On clinical grading of 172 limbs, $\mathbf{4 5 / 1 7 2}$ were Grade 0, 62 were graded as I, 21 in Grade II, and 44 were Grade III (Table 4a). The distribution of limbs on visual grading is detailed in Table $4 b$ as shown in Fig. 4.

Based on qualitative lymphoscintigraphy, the clinical grade was upgraded (Table 5 ). Out of $\mathbf{4 5}$ clinically normal limbs, only 7 (15.5\%) limbs were normal on qualitative lymphoscintigraphy. A total of 38/45 (84.5\%) limbs were upgraded to higher grades - 32 (71.1\%) to Grade I, 4 (8.8\%) to Grade II and 2 (4.4\%) to Grade III. Similarly, 19 limbs with Grade I lymphedema on clinical examination showed evidence of higher grade on qualitative lymphoscintigraphy. Most of them were upgraded to Grade II. Only 9/21 (42.8\%) clinically Grade II limbs were upgraded to Grade III on visual analysis.

Ilio-inguinal nodal uptake percentage was calculated for all the 172 limbs. According to the range of percentage nodal uptake obtained from the control group, the percentage nodal uptake in $\mathbf{1 7 2}$ limbs was evaluated. The limbs with percentage nodal uptake less than the normal range (19.72 to $53.32 \%$ ) of the control group were considered abnormal and those with uptake within the range and above were considered normal. Out of 172 limbs, 114 $(66.2 \%)$ limbs were categorized as abnormal, $58(33.8 \%)$ as normal by quantitative lymphoscintigraphy.

\section{Comparison of results with qualitative and quantitative analysis}

Out of 172 limbs, $114(66.2 \%)$ limbs were shown as abnormal by quantitative method. $57 / 80(71.25 \%)$ limbs of Grade I, 19/24 (79.16\%) of Grade II, 34/45 (75.55\%) of Grade III and 4/4 limbs of Grade IV were abnormal on quantitative analysis (Table 6 ). Only 96/172 (55.8\%) were abnormal on both qualitative and quantitative lymphoscintigraphy.

A significant difference was found between the two techniques (qualitative and quantitative methods) in detecting lymphedema, with qualitative method being more accurate than quantification by nodal uptake percentage (crosstabs and Chi square test, $\mathrm{p}=0.004)($ Table 7). 


\section{TABLE 5}

5a: Upgradation of clinical Grade 0 by qualitative analysis

\begin{tabular}{|l|c|c|}
\hline Clinical grade & $\begin{array}{c}\text { Qualitative } \\
\text { lymphoscintigraphic grade }\end{array}$ & Number of grades (out of 45) \\
\hline Grade 0 & Normal & $7(15.5 \%)$ \\
\hline Grade 0 & Grade I & $32(71.1 \%)$ \\
\hline Grade 0 & Grade II & $4(8.8 \%)$ \\
\hline Grade 0 & Grade III & $2(4.4 \%)$ \\
\hline
\end{tabular}

5b: Upgradation of clinical Grade 1 and 2 by qualitative analysis

\begin{tabular}{|l|c|c|}
\hline Clinical grade & $\begin{array}{c}\text { Qualitative } \\
\text { lymphoscintigraphic grade }\end{array}$ & Number of limbs \\
\hline Grade 1 & Grade II & $12(19.3 \%)$ \\
\hline Grade 1 & Grade III & $07(11.2 \%)$ \\
\hline Grade 2 & Grade III & $09(42.8 \%)$ \\
\hline
\end{tabular}

\begin{tabular}{|c|c|c|}
\hline \multicolumn{3}{|c|}{ TABLE 6 } \\
Correlation Between Visual Grading and Nodal Uptake Percentage \\
\hline $\begin{array}{c}\text { Qualitative } \\
\text { lymphoscintigraphic grade }\end{array}$ & Number of limbs & $\begin{array}{c}\text { Number of abnormal limbs } \\
\text { on quantitative scintigraphy }\end{array}$ \\
\hline Grade I & & $57(71.25 \%)$ \\
\hline Grade II & 80 & $19(79.16 \%)$ \\
\hline Grade III & 24 & $34(75.55 \%)$ \\
\hline Grade IV & 45 & $04(100 \%)$ \\
\hline
\end{tabular}

\begin{tabular}{|c|c|c|c|c|}
\hline \multicolumn{5}{|c|}{$\begin{array}{l}\text { TABLE } 7 \\
\text { Analysis with Combined Qualitative and Quantitative Methods }\end{array}$} \\
\hline & \multicolumn{3}{|c|}{ QUALITATIVE } & Total \\
\hline \multirow{3}{*}{ QUANTITATIVE } & & Normal & Abnormal & \\
\hline & Normal & 1 & 57 & 58 \\
\hline & Abnormal & 18 & 96 & 114 \\
\hline Total & & 19 & 153 & 172 \\
\hline
\end{tabular}


TABLE 8

Grade-Wise Correlation with Nodal Uptake Percentage

\begin{tabular}{|c|c|c|c|}
\hline $\begin{array}{c}\text { Qualitative } \\
\text { lymphoscintigraphic grade }\end{array}$ & $\begin{array}{c}\text { Number of } \\
\text { limbs }\end{array}$ & $\begin{array}{c}\text { Mean percentage } \\
\text { nodal uptake (\%) }\end{array}$ & Standard deviation \\
\hline Normal & 19 & 36.64 & 11.38 \\
\hline Grade I & 80 & 16.67 & 16.05 \\
\hline Grade II & 24 & 13.95 & 11.25 \\
\hline Grade III & 45 & 11.56 & 10.99 \\
\hline Grade IV & 04 & 0.075 & 0.15 \\
\hline
\end{tabular}

Grade-wise definition of mean ilio-inguinal nodal uptake percentage

The corresponding mean percentage nodal uptake in the grades of lymphedema by qualitative lymphoscintigraphy was assessed. The corresponding nodal uptake percentage for each grade is as detailed in Table 8 .

Applying an ANOVA analysis, the difference of percentage nodal uptake in between groups is found to be statistically significant $(p=0.03)$. The mean percentage nodal uptake in normal limbs is $36.64 \%$ which reduces to $16.67 \%$ in Grade I, $13.95 \%$ in Grade II, $11.56 \%$ in Grade III, and $0.07 \%$ in Grade IV. As the severity of lymphedema increases which is seen by increasing grade on qualitative lymphoscintigraphy, there is a fall in the nodal uptake (Fig. 5 ).

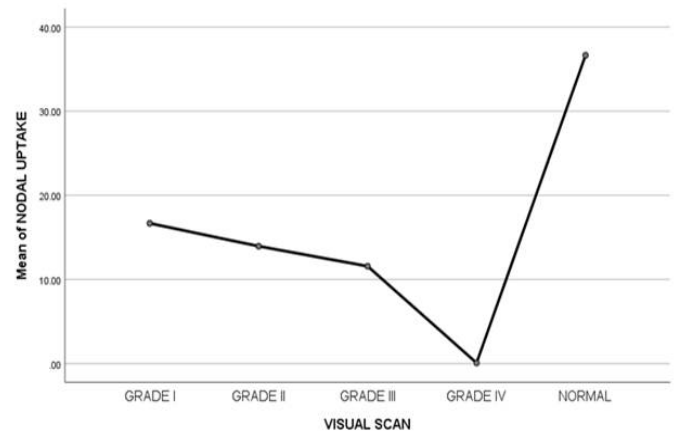

Fig. 5. Mean plot displaying falling nodal uptake percentage as severity of grade increases.
Defining a cut off percentage nodal uptake to differentiate between normal and abnormal limbs

Using receiver operating curve (ROC), the cut off percentage nodal uptake which can differentiate between normal and abnormal limbs was derived to be $22.18 \%$ with a sensitivity of $100 \%$ and specificity of $79.82 \%$. ROC had a maximal area under curve of 0.904 (Fig. 6) indicating that quantitative lymphoscintigraphy with percentage nodal uptake is useful in diagnosing lymphedema.

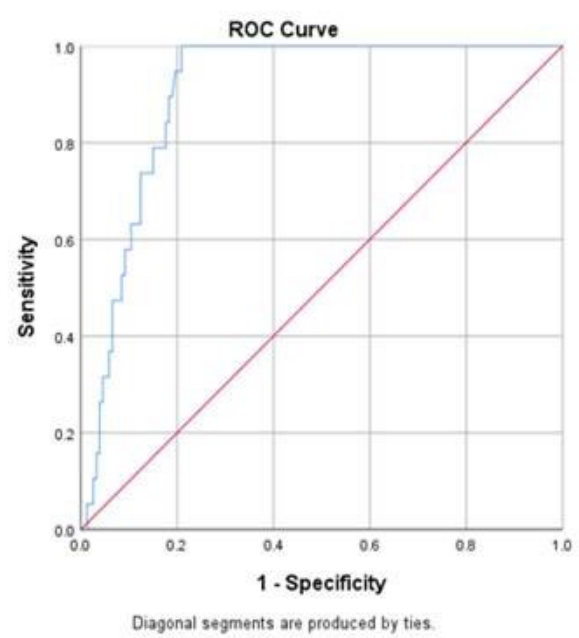

Fig. 6. Receiver operator curve analysis (ROC) establishing cut-off nodal uptake percentage to differentiate normal from abnormal limbs. 


\begin{tabular}{|c|c|c|c|}
\hline \multicolumn{5}{|c|}{$\begin{array}{c}\text { TABLE 9 } \\
\text { Qualitative and Quantitative Analysis of Cases with Unilateral } \\
\text { Lymphedema by Lymphoseintigraphy }\end{array}$} \\
\hline Clinical grade & $\begin{array}{c}\text { Qualitative } \\
\text { lymphoscintigraphic } \\
\text { grade }\end{array}$ & $\begin{array}{c}\text { Number of } \\
\text { limbs }\end{array}$ & $\begin{array}{c}\text { Mean percentage nodal } \\
\text { uptake (\%) }\end{array}$ \\
\hline Grade 0 & Normal & $02(10 \%)$ & $24.25 \%$ \\
\hline Grade 0 & Grade I & $14(70 \%)$ & $16.36 \%$ \\
\hline Grade 0 & Grade II & $03(15 \%)$ & $05.97 \%$ \\
\hline Grade 0 & Grade III & $01(05 \%)$ & $\begin{array}{c}33.63 \% \\
\text { (observed value for 1 } \\
\text { patient) }\end{array}$ \\
\hline
\end{tabular}

\section{Analysis of results of lymphoscintigraphy in patients with unilateral lymphedema}

Out of 86 patients, 20 patients had presented with unilateral lymphedema (out of 40 limbs, 20 were normal and 20 abnormal). Out of the 20 normal limbs which were clinically Grade 0, 18/20 (90\%) limbs were found to have altered lymphatic drainage and $2 / 20$ showed normal lymphatic drainage pattern on qualitative lymphoscintigraphy (Table 9). Out of these 18 limbs which were upgraded based on the visual qualitative analysis, only $2 / 18(11 \%)$ had normal nodal uptake percentage.

\section{DISCUSSION}

Lymphoscintigraphy is considered the gold-standard investigation to establish the diagnosis of lymphedema and to visualize small and large caliber vessels $(5,7)$. The sensitivity of lymphoscintigraphy to ascertain lymphedema ranges from $77-78 \%$ with a specificity of $100 \%$ (2). Diagnosis of lymphedema is done essentially by clinical history and physical examination, and lymphoscintigraphy is often employed for confirmation of the diagnosis. It serves not only as a diagnostic tool, but also can provide a global impression for the management of the patient.

Qualitative lymphoscintigraphy images the morphology of the lymphatic system whereas quantitative lymphoscintigraphy aims to measure lymphatic flow and is regarded to be a sensitive way of diagnosing lymphatic impairment. However, quantification is not routinely performed. Methods of quantification include the ilio-inguinal lymph nodal uptake and the rate of clearance of tracer from the injection site. Both the methods are technically difficult (6). There are also measures of time to the node and lymphatic speed. All methods have reproducibility issues and have not been standardized.

In this study, $85.4 \%$ clinically normal limbs were upgraded to higher grades by lymphoscintigraphy, $71 \%$ of them to Grade I showing multiple tortuous channels or delay to flow of tracer on lymphoscintigraphy. This supports a prior study undertaken by our institution previously by K Shilpa et al (6) who demonstrated an upgrading of clinically normal limbs in $61 \%$ of patients in the study of 424 patients.

Out of 20 patients presenting with unilateral edema, 18/20 (90\%) of contralateral normal looking limbs on clinical examination were upgraded on qualitative lymphoscintigraphy suggesting that in cases of unilateral lymphedema, even the contralateral limb will have some sub-clinical non-apparent lymphedema. Diagnosing lymphedema early can provide early intervention resulting in reduced 
chances of disability in the future. Similarly, a previous study of 418 patients by Shelley $S$ et al (7), concluded that lymphoscintigraphy aids in the corrective management of lymphedema by diagnosing subclinical lymphatic dysfunction. Nevertheless, on quantification, a significant overlap was found in normal and abnormal limbs with only $2 / 18$ limbs showing abnormal nodal uptake percentage. It should be noted that our study included patients from a filarial area and this percentage of contralateral lymphatic disturbance may not apply to all populations in other settings.

The sensitivity of lymphoscintigraphy obtained from this study for qualitative and quantitative lymphoscintigraphy techniques are $88.9 \%$ and $66.3 \%$, respectively. Weissleder and Weissleder (8) in 1988 conducted a study of 308 lower limbs with lymphedema. They reported a sensitivity of $68 \%$ by qualitative analysis which improved to $100 \%$ on combining results of quantification. They utilized Tc99m-human serum albumin subcutaneously in the first interdigital space of feet and undertook a visual analysis as well as estimated the rate of clearance of tracer from the injected site. Firstly, there is a difference in the technique of quantification employed in both the studies. Moreover, the radiotracers used also differ along with the route of administration and in size ( 2 factors resulting in faster drainage with Sb2S3 than with HSA nanocolloids). In addition, there has been a huge advancement in the gamma camera apparatus and techniques over the time, however this has not resulted in better quantification.

Roberta Maria Dalia et al (9) performed a study in 154 patients of lymphedema using Tc99m-dextran injected in all four interdigital spaces intradermally. Both rate of clearance of tracer from the injection site and lymph nodal uptake were incorporated for quantification (9). They concluded that qualitative lymphoscintigraphy performed sub-optimally in cases of milder lymphedema (Grades I and II) where quantification demonstrated a superior outcome. The reported sensitivity of qualitative analysis was $61 \%$ in milder grades of lymphedema. Likewise, qualitative analysis by visual interpretation in Grades I and II performed in this study resulted in a sensitivity of $60 \%$. On the contrary, quantitative analysis showed a sensitivity of only $44 \%$ in these milder grades. However, the difference between the mean nodal uptake percentage corresponding to grades I and II was found to be statistically significant $(\mathrm{p}=\mathbf{0 . 0 3})$.

The nodal uptake percentage corresponding to normal, Grade I, II, III, and IV was found to be $36.64 \%, 16.67 \%, 13.95 \%$, $11.56 \%$ and $0.075 \%$, respectively, in this study. A significant difference in the uptake percentage was noted in between the normal and abnormal limbs as well as in between grades $(\mathrm{p}=0.03)$. However, quantitative lymphoscintigraphy with Tc99m-nanocolloid employing tracer absorption was found to be unreliable in higher grades of lymphedema (Grades III and IV) in a previous study devised in our institution of 32 patients with lymphedema by Shilpa $\mathrm{K}$ et al (6). This is likely due to the difference in technique of quantification since they used rate of clearance of tracer from injection site.

When considering quantitative analysis alone for evaluation of lymphedema, the sensitivity and specificity of the test was very low in comparison with a combined evaluation using both quantitative and qualitative examination. This is in contrast to the study done by Nganga and Makhdomi (10) who proved that quantification by nodal uptake is a reliable method for quantification. Multiple studies have been described investigating quantitative lymphoscintigraphy as a sensitive approach for diagnosis. However, the results of this study are contradictory, and this difference in results might be a reflection of the diversity in the population which had been studied.

For differentiating between normal and abnormal limbs using only quantitative method, a genuine cut-off percentage is needed. Through ROC analysis, taking qualitative method as gold standard, a cut-off percentage of $22.18 \%$ was defined by us with a sensitivity of $100 \%$ and specificity of $79.82 \%$. ROC had a maximal area under curve of 0.904 indicating that quantitative lymphoscintigraphy with percentage nodal uptake is useful in diagnosing lymphedema. Similarly, Nganga $(4,10)$ in his study defined a cut-off percentage of $19.7 \%$ 


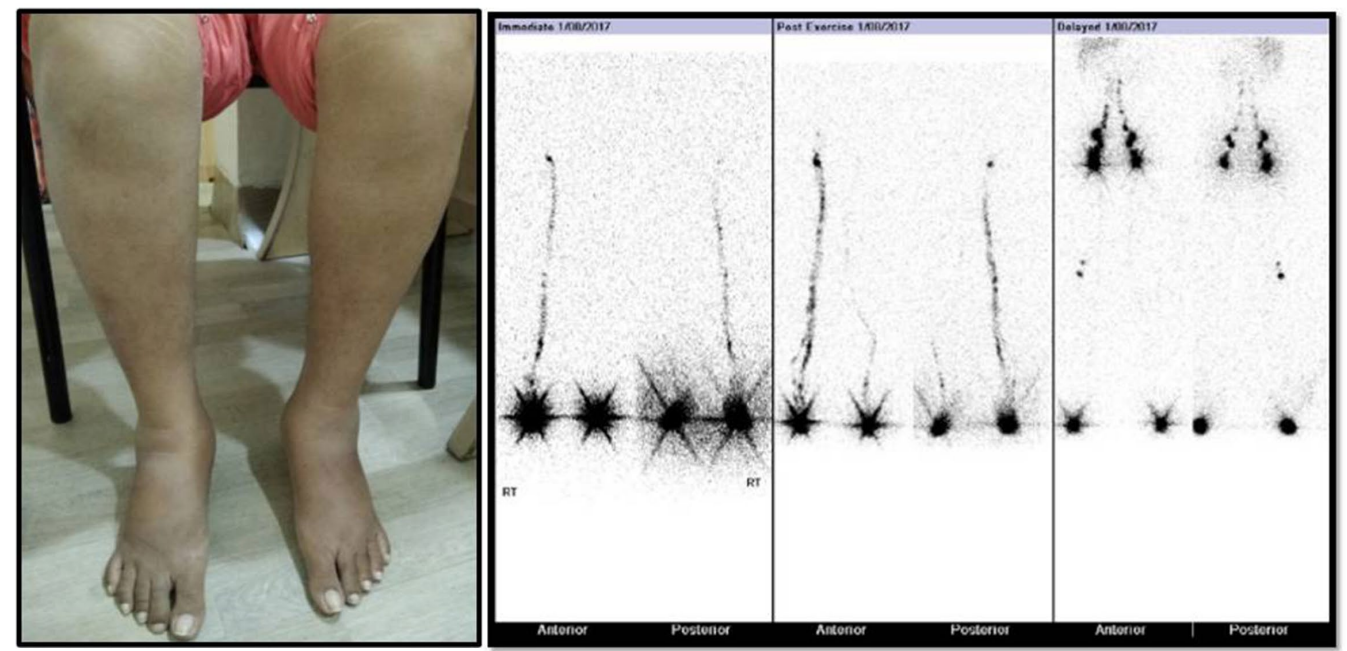

Fig. 7. a) 28 years-old male who presented with genital swelling since 3 years with no significant edema in the lower limbs and a bilateral clinical Grade 0. b) Lymphoscintigraphic findings in the right limb shows lymphatic drainage through a single lymphatic channel with visualization of inguinal nodes in the immediate images. Deep group of nodes (popliteal) is noted in the delayed images producing a Grade II assessment. The left limb demonstrates delay to flow of tracer with visualization of inguinal nodes in the delayed images producing a Grade I assessment. The nodal uptake percentage is for the right is 52\% and for the left $17 \%$.
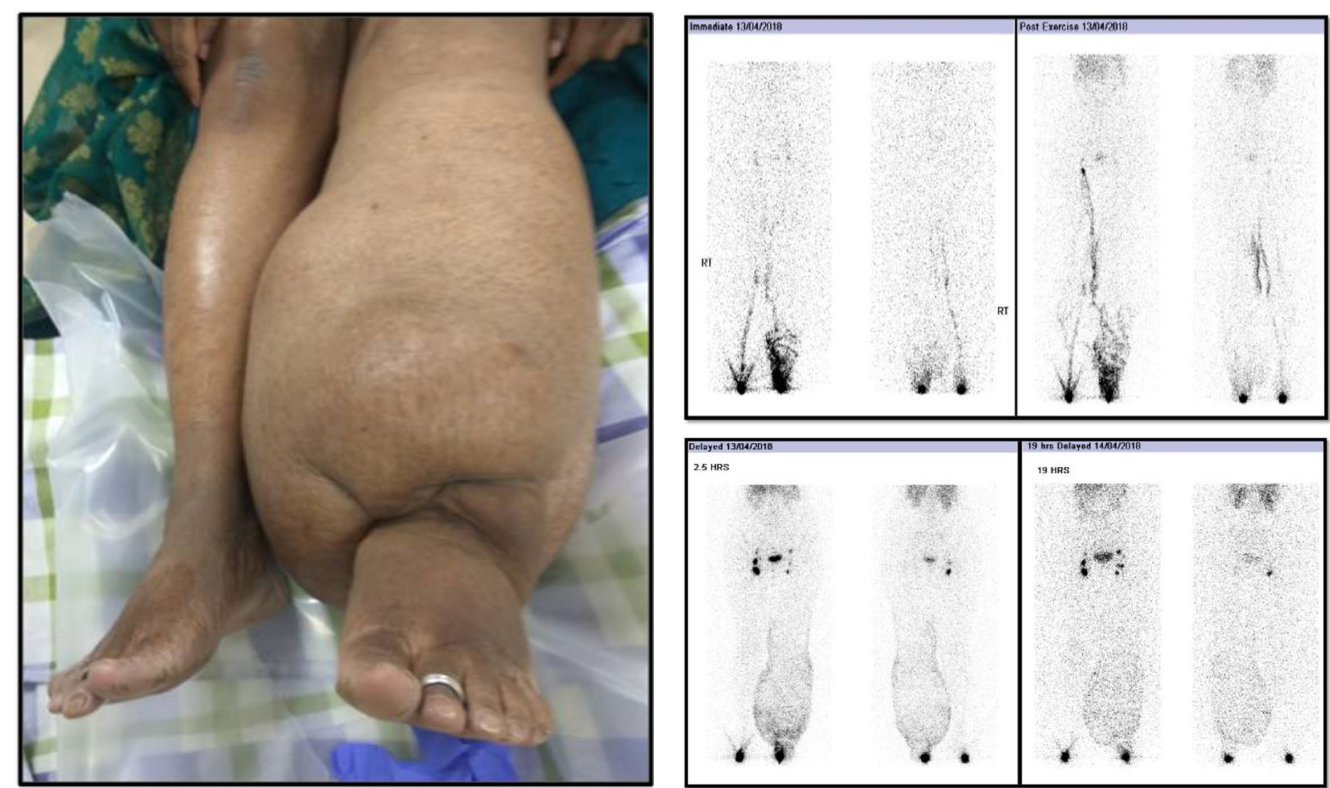

Fig. 8. a) 49 year-old female, presented with left lower limb swelling since 10 years of age. Clinically edema was non pitting and irreversible and to the level of knees with a clinical Grade III. b) : Lymphoscintigraphic findings immediate and post exercise images on the right limb shows delayed flow of tracer through multiple lymphatic channels with visualization of inguinal nodes in post exercise images producing a Grade I assessment. The left limb shows multiple lymphatic channels with delayed visualization of inguinal nodes and evidence of dermal backflow in the leg region producing a Grade III assessment. The nodal uptake percentage for the right is $13.9 \%$ and for the left 6.7\%.c) 3 and 19 hrs delayed images show dermal backflow in the leg region in left limb with visualization of inguinal nodes in the delayed images. 

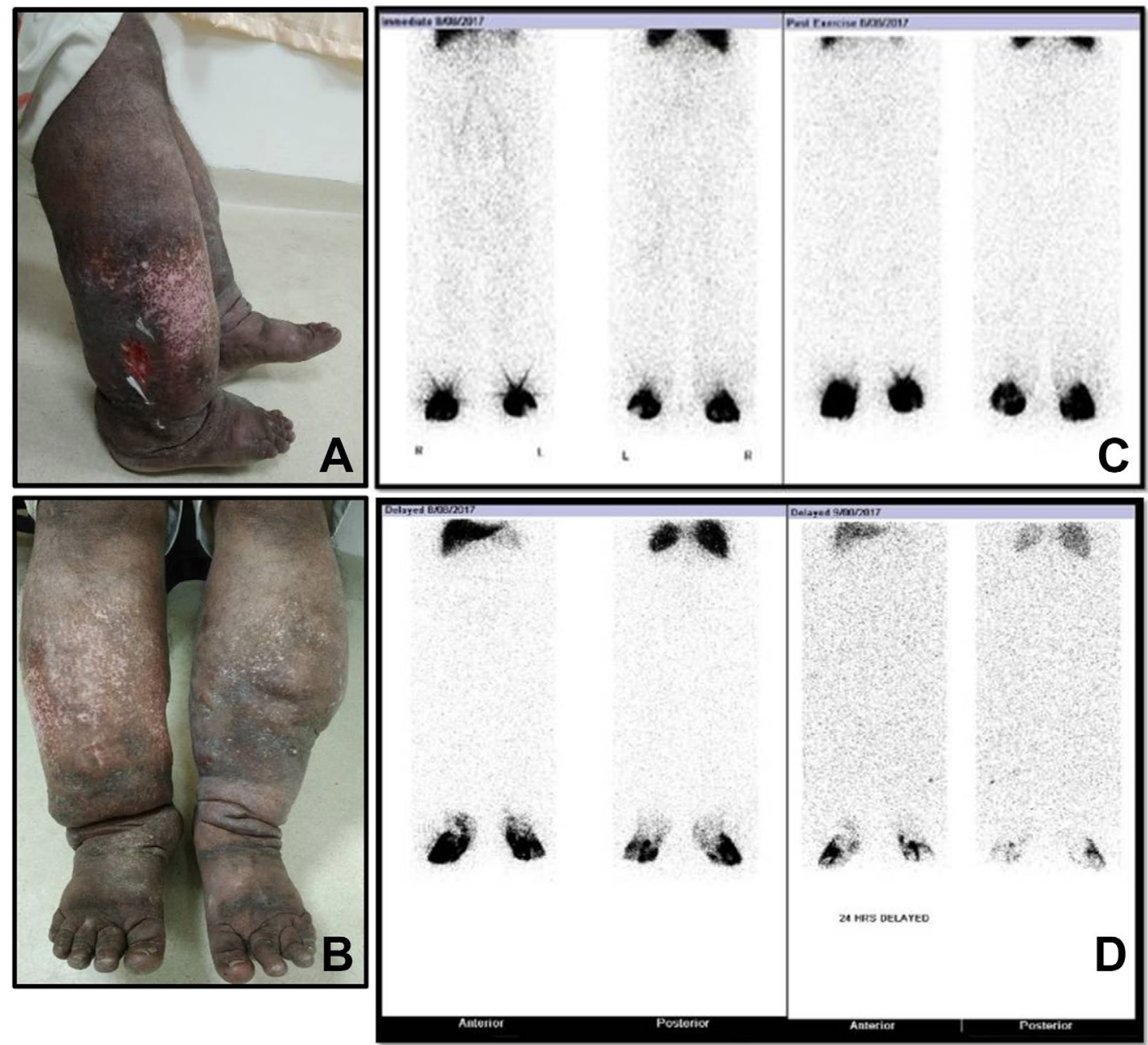

Fig. 9. a,b) 56 year-old male presented with bilateral lower limb swelling. Clinically edema was non-pitting, irreversible associated with nodular skin thickening, plaquing, desquamation, and ulceration on the right leg laterally (bilateral clinical Grade III). c) Lymphoscintigraphy immediate and post exercise sweep images show no demonstrable lymphatic channels or nodes in bilateral lower limbs. Minimal dermal backflow can be seen in the foot region of both the limbs. d) Delayed images at 3 hrs and 24 hrs do not show any lymphatic channels or inguinal nodes producing a lymphoscintigraphic grading of Grade IV.

in normal limbs as compared to 5.5 in abnormal limbs. They speculated that ilioinguinal nodal uptake is a reliable parameter in quantitative analysis and can be used to differentiate normal from abnormal limbs where qualitative analysis is equivocal in a group of 52 patients with long standing lower limb lymphedema. In this study, we applied the quantification of accumulation of tracer in nodes by calculating the nodal uptake percentage by using the formula devised by
Nganga and colleagues $(4,10)$. The difference between the two studies is that Nganga and colleagues drew the ROIs in $1.5 \mathrm{hr}$ images whereas we did at 3 hours.

The uptake percentage in a normal limb as found by us was 19.7 to $53.3 \%$ with SD of 8.4. In spite of the differences in procedure protocol, the findings were found to be similar with Keramida et al (11) who quantified the ilio-inguinal nodal uptake using Tc99mnanocolloid in a study of 102 patients. The 

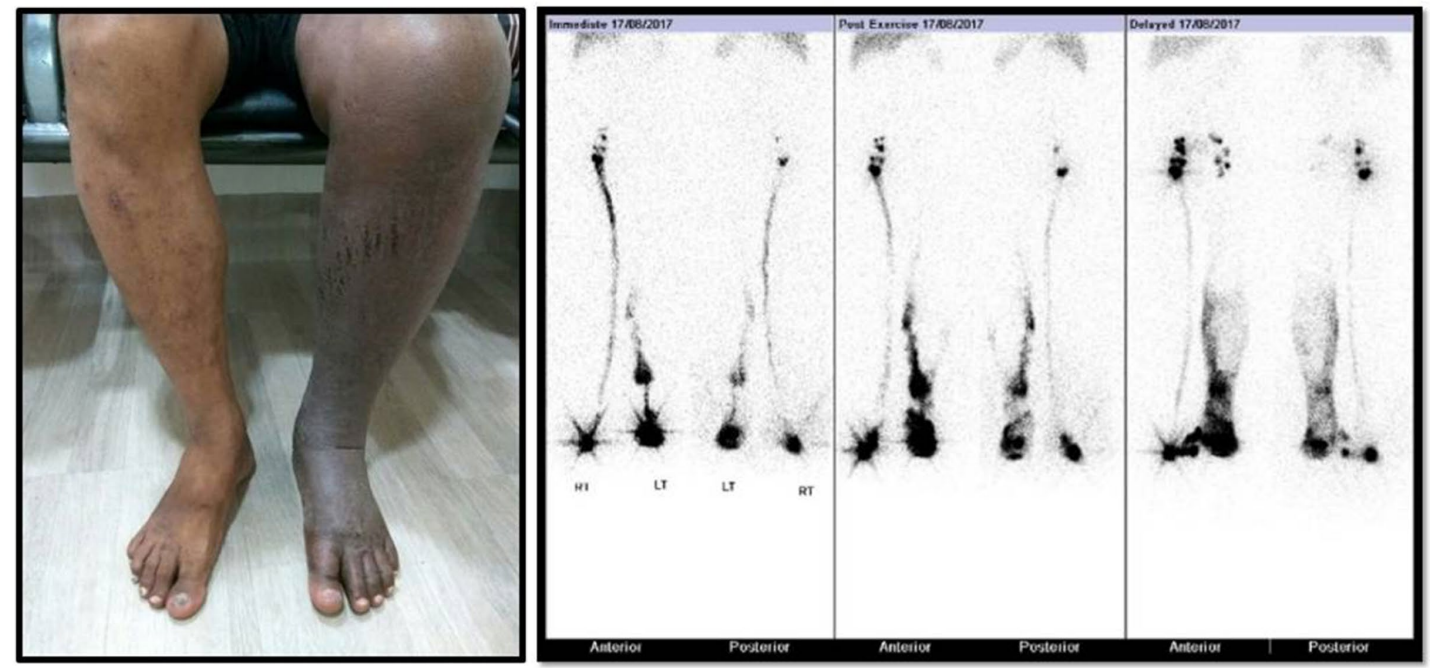

Fig. 10. a) 61 year-old male, presented with left lower limb swelling. Left limb showed non-pitting, irreversible edema with tense skin associated with hyperpigmentation (clinical Grade III). Clinically right limb was normal (clinical Grade 0). b) Lymphoscintigraphic findings on the right limb shows flow of tracer through single lymphatic channel with visualization of inguinal nodes in the immediate images. No evidence of tracer stasis or deep nodes and dermal backflow is seen (normal). The left limb demonstrates delay in flow of tracer through multiple lymphatic channels with visualization of inguinal nodes in the delayed images. There is evidence of dermal backflow in the leg region (Grade III). The nodal uptake percentage for the right is 22\% and for the left is $0.6 \%$.

normal nodal uptake percentage he had obtained was 7.9 to $38 \%$ with a high standard deviation. However, the route of injection was subcutaneous and formulae used and acquisition protocol were different in comparison with this study.

Though the quantitative method increases the diagnostic sensitivity of lymphoscintigraphy, there is a need for the standardization of the imaging protocol based on radiotracer and exercise regimen, even more important than in qualitative studies to enable the establishment of 'normal' values and define abnormalities. It is also important that any such exercise regimen is manageable for patients whose mobility is limited by their lymphedema (2).

The limitations of this study lie in the facts that the rate of clearance of tracer from injection site was taken as constant for all patients, while practically it differs from patient to patient. Delayed imaging was done at 3 hours, before which the pharmacokinetics and dynamics cannot be commented upon.
Also, the BMI of the patient and general body habitus (lean, overweight, and obese) have not been considered, which can play a major role in the attenuation of iliac nodes. Lastly, the number of controls incorporated for the study during the study period (which was limited pertaining to the course of 2 years) was very small, thus the normal nodal uptake percentage obtained might not be reliable and might have caused discordance between the visual and quantitative analysis of the limbs.

\section{CONCLUSION}

Radionuclide lymphoscintigraphy is an unfailing diagnostic modality to ascertain lymphedema. Qualitative lymphoscintigraphy is a more accurate and sensitive technique as compared to quantitative methods with nodal tracer accumulation in establishing the diagnosis. Though quantitative analysis with nodal uptake percentage provides objective criteria to diagnose lymphedema, it can only act as an adjunct to the qualitative method 
and cannot replace it. Lymphoscintigraphy can establish subclinical lymphatic dysfunction and can aid in preventive management of lymphedema. Standardization of procedure for quantitative lymphoscintigraphy is needed along with an approach which combines both rate of clearance of tracer from injection site and nodal uptake for quantification.

\section{CONFLICT OF INTEREST AND DISCLOSURE}

The authors declare no competing financial interests exist.

\section{REFERENCES}

1. Andrzej, S, S William, H William, R Stanley: The third circulation: Radionuclide lymphoscintigraphy in the evaluation of lymphedema. J. Nucl. Med. 44 (2003), 43-57.

2. Keeley, V: The use of lymphoscintigraphy in the management of chronic oedema. J. Lymphoedema 1 (2006), 42-57.

3. Burnand, KM, DM Glass, PS Mortimer, et al: Lymphatic dysfunction in apparently clinical normal contralateral limbs of patients with unilateral lower limb swelling. Clin. Nucl. Med. 37 (2012), 9-13.

4. Nganga, EC, G Samuel, M Khalid: Lower limb lymphoscintigraphy patterns among patients with lower limb lymphedema: a pictorial essay. Clin. Translat. Imaging 6 (2018), 135-142.

5. Executive Committee of the International Society of Lymphology. The diagnosis and treatment of peripheral lymphedema: 2020
Consensus Document of the International Society of Lymphology. Lymphology 53 (2020), 3-19.

6. Shilpa, K, M Indirani, S Shelley, G Manokaran: Lymphoscintigraphy as an imaging modality in lymphatic system. Apollo Medicine 7 (2010), 205-210.

7. Shelley, S, G Manokaran, M Indirani, et al: Lymphoscintigraphy as a diagnostic tool in patients with lymphedema of filarial origin. Lymphology 39 (2006), 69-75.

8. Weissleder, $\mathrm{H}, \mathrm{R}$ Weissleder: Lymphedema: Evaluation of qualitative and quantitative lymphoscintigraphy in 238 patients. Radiology 167 (1988), 729-735.

9. Dalia, R, G Ribeiro, Martins et al: Qualitative and quantitative lymphoscintigraphy in the evaluation of lower limbs lymphedema. Brazilian Arch. Biol. Technol. 48 (2005), 159-162.

10. Nganga, EC, K Makhdomi: Comparison of quantitative analysis to qualitative analysis for interpretation of lower-limb lymphoscintigraphy. World J. Nucl. Med. 18 (2019), 36.

11. Keramida, G, N Winterman, E Wroe, et al: Importance of accurate ilio-inguinal quantification in lower extremity lymphoscintigraphy. Nucl. Med. Comm. 38 (2017), 209-214.

\author{
Dr. Shelley Simon, DRM, DNB \\ Department of Nuclear Medicine and \\ PET/CT \\ Apollo Hospitals \\ 21 Greams Lane \\ Chennai, Tamil Nadu, India \\ E-mail: shelleysimon@rediffmail.com
}

